\title{
Dynamics of the Delayed Business Cycle Model with Expectation and General Saving Function
}

\author{
Ratchata Utama, Ekkachai Kunnawuttipreechachan* \\ Department of Mathematics, Faculty of Applied Science, \\ King Mongkut's University of Technology North Bangkok, Bangkok 10800, Thailand
}

\begin{abstract}
Cite This Paper in the following Citation Styles
(a): [1] Ratchata Utama, Ekkachai Kunnawuttipreechachan, "Dynamics of the Delayed Business Cycle Model with Expectation and General Saving Function," Universal Journal of Accounting and Finance, Vol.9, No.2, pp. 210-216, 2021. DOI: 10.13189/ujaf.2021.090210.

(b): Ratchata Utama, Ekkachai Kunnawuttipreechachan, (2021). Dynamics of the Delayed Business Cycle Model with Expectation and General Saving Function. Universal Journal of Accounting and Finance, 9(2), 210-216. DOI: 10.13189/ujaf.2021.090210.
\end{abstract}

Copyright $@ 2021$ by authors, all rights reserved. Authors agree that this article remains permanently open access under the terms of the Creative Commons Attribution License 4.0 International License

\begin{abstract}
In this paper, we study a class of business cycle models related to the relationship between the gross product and the capital stock. The model is extended by adding general terms of the investment and the saving functions. In addition, a time delay which is represented an expectation and time lag for investment is also added into the model. The aim for this work is to find the sufficient conditions in which the model's solution is periodic. We study the existence of unique positive equilibrium. Next, the linearization method is used to analyze local behavior of the model. In addition, by considering time delay as a bifurcation parameter, we investigate sufficient conditions for the existence of a Hopf bifurcation of the model. Our results are applied to represent conditions for an occurrence of business cycles, which can happen in many business situations. Finally, some numerical simulations are illustrated to support our theoretical results.
\end{abstract}

Keywords Business Cycle, Stability Analysis, Hopf Bifurcation, Time Delay

\section{Introduction}

Business cycle is an economic behavior which is fluctuations of macroeconomic variables caused by uncertainty of business systems. There are many methods to analyze behaviors and factors for the occurrence of a business cycle. Mathematical method is one of the efficiency methods for the analysis of business and economic problems. Hence this paper is focused on the usage of mathematical theorems and methods to study factors that cause the business cycle related to the relation between the gross product and the capital stock.

The development of mathematical models for business cycle are stated as follows. In 1935, Kalecki [1] explained concepts of consumption functions and multipliers in statistical analysis for study macroeconomics. In 1940, Kaldor [2] presented mechanisms of cyclic fluctuations for a business cycle model with nonlinear investment and saving functions, which are important in economical analysis. The publications of Kaldor and Kalecki are based for modeling of trade cycle for investment and saving functions. In 1971, Chang and Smyth [3] introduced the model for business cycle as

$$
\begin{aligned}
& \frac{d Y(t)}{d t}=\alpha[I(Y(t), K(t))-S(Y(t), K(t))], \\
& \frac{d K(t)}{d t}=I(Y(t), K(t)),
\end{aligned}
$$

where $Y$ refers to the gross product, $K$ represents the capital stock, $I$ represents the investment function, $S$ is the saving function, and $\alpha$ is an adjustment coefficient in the goods market. Note that model (1) is usually called the Kaldor-Kalecki model to explain business cycle related to the gross products and the capital stocks.

In 1994, the Kaldor-Kalecki model (1) was improved by Grasman and Wentzel [4] by adding a linear saving function on the change rate of the capital stock. Therefore, the change of capital stocks is a difference between an investment function and a depreciation rate with the capital stock. The model in [4] is assumed that the saving function is linear and depends only on the capital stock, i.e. $S(Y, K)=\gamma K$. Then the modified 
model of (1) becomes

$$
\begin{aligned}
& \frac{d Y(t)}{d t}=\alpha[I(Y(t), K(t))-\gamma Y(t)], \\
& \frac{d K(t)}{d t}=I(Y(t), K(t))-\beta K(t) .
\end{aligned}
$$

Here $\beta$ is the depreciation rate of the capital stock, and $\gamma \in$ $(0,1)$ is a constant.

In general, delay differential equations are used to model many social and scientific behaviors, such as in environmental and epidemical problems (see, for examples, in [5-9]). The time delays are usually represented time lag or waiting time between state variables of models.

For business cycle model, time delays are usually added in many literatures, such as in [10-14]. In [15] Kaddar and Talibi added a time delay $\tau$ into the gross product and the capital stock. In their works, the time delay represents time for decision changing in the capital stock due to the past investment decisions. Therefore, system (2) becomes

$$
\begin{aligned}
& \frac{d Y(t)}{d t}=\alpha[I(Y(t), K(t))-S(Y(t), K(t))] \\
& \frac{d K(t)}{d t}=I(Y(t-\tau), K(t-\tau))-\beta K(t) .
\end{aligned}
$$

In this work, we study a class of business cycle model, which called the Kaldor-Kalecki business cycle model with time delays and an impact of expected capital stock on the current investment decisions. Moreover, the expected capital stock $K(t+\tau)$ is added into (3). Then, the revised model is

$$
\begin{aligned}
& \frac{d Y(t)}{d t}=\alpha[I((Y(t), K(t+\tau))-S(Y(t))], \\
& \frac{d K(t)}{d t}=I(Y(t-\tau), K(t-\tau))-\beta K(t) .
\end{aligned}
$$

The main aim of this work is to investigate the sufficient conditions for the existence of unique positive equilibrium of (4). We analyze the local stability and determine the conditions for an exhibition of Hopf bifurcation. Finally, we illustrate numerical simulations to support the analytical results.

\section{The Model Analysis}

In this section, we investigate conditions for the existence of unique positive equilibrium. Further, we analyze the dynamical behavior of (4) about the positive equilibrium. We also find the conditions for an occurrence of a Hopf bifurcation, which shows that there exists a cycle in business phenomena.

\subsection{Existence of unique positive equilibrium}

Firstly, we investigate conditions for existence of unique positive equilibrium $\mathbf{E}^{*}=\left(Y^{*}, K^{*}\right)$ of (4). To find the business equilibrium of (4), we set $Y^{\prime}(t)=K^{\prime}(t)=0$ and $Y(t)=Y^{*}, K(t)=K^{*}$. In addition, we assume that the general investment function $I(Y, K)$ and the saving function $S(Y)$ must satisfy the following assumptions:
$\left(A_{1}\right) S(0)=0$ and $I(0,0)>0$,

$\left(A_{2}\right) \frac{\partial I}{\partial Y}-\frac{d S}{d Y}+\frac{1}{\beta} \frac{\partial I}{\partial K}<0$ for all $(Y, K) \in \mathbb{R}^{2}$,

$\left(A_{3}\right)$ there exist constants $L \geq 0$ and $\bar{p} \geq 0$ such that $|I(Y, K)+\bar{p} K| \leq L$, for all $Y, K \in R$.

Theorem 1. If assumptions $\left(A_{1}\right)-\left(A_{3}\right)$ hold, then model (4) has unique positive equilibrium $\boldsymbol{E}^{*}=\left(Y^{*}, K^{*}\right)$, where $K^{*}=\frac{1}{\beta} S\left(Y^{*}\right)$ and $Y^{*}$ is the unique solution of $I\left(Y^{*}, \frac{1}{\beta} S\left(Y^{*}\right)\right)-S\left(Y^{*}\right)=0$.

Proof. The equilibrium $\mathbf{E}^{*}=\left(Y^{*}, K^{*}\right)$ of the model (4) can be analyzed by solving the system

$$
\begin{aligned}
\alpha\left[I\left(Y^{*}, K^{*}\right)-S\left(Y^{*}\right)\right] & =0, \\
I\left(Y^{*}, K^{*}\right)-\beta K^{*} & =0 .
\end{aligned}
$$

From (5) the equilibrium must satisfy equations

$$
I\left(Y^{*}, K^{*}\right)=S\left(Y^{*}\right)
$$

and

$$
K^{*}=\frac{1}{\beta} S\left(Y^{*}\right) .
$$

Next, substitute $K^{*}$ from (7) into (6). Thus, we have

$$
I\left(Y^{*}, \frac{1}{\beta} S\left(Y^{*}\right)\right)-S\left(Y^{*}\right)=0 .
$$

Define the function $f$ as a function of the capital stock $Y$ by

$$
f(Y)=I\left(Y, \frac{1}{\beta} S(Y)\right)-S(Y),
$$

where $Y \in[0,+\infty)$.

From $\left(A_{1}\right)$, we have $f(0)=I(0,0)>0$. From $\left(A_{2}\right)$, $f^{\prime}(Y)=\frac{\partial I}{\partial Y}-\frac{d S}{d Y}+\frac{1}{\beta} \frac{\partial I}{\partial K}<0$. According to $\left(A_{3}\right)$, we can obtain that $\lim _{Y \rightarrow \infty} f(Y)=-\infty$.

Thus, model (4) has unique positive equilibrium $\mathbf{E}^{*}=$ $\left(Y^{*}, K^{*}\right)$, where $K^{*}=\frac{S\left(Y^{*}\right)}{\beta}$, and $Y^{*}$ is the positive solution, which is the solution of (8).

\subsection{Stability analysis and existence of Hopf bi- furcation}

From the business cycle model (4), the positive equilibrium $\mathbf{E}^{*}=\left(Y^{*}, K^{*}\right)$ is obtained by Theorem 1 . In this part, we will analyze stability of (4) near the equilibrium $\mathbf{E}^{*}$. We firstly transform the model with an expected capital stock to the current investment decision for stability analysis. Let

$$
Z(t)=K(t+\tau)
$$


Next, substitute $Z(t)$ from (9) into (4), then we have

$$
\begin{aligned}
& \frac{d Y(t)}{d t}=\alpha[I(Y(t), Z(t))-S(Y(t))], \\
& \frac{d Z(t)}{d t}=I(Y(t), Z(t-\tau))-\beta Z(t) .
\end{aligned}
$$

To investigate the local asymptotic stability, we consider stability of (10) about the equilibrium $\mathbf{E}^{*}=\left(Y^{*}, Z^{*}\right)$. Note that from (9), we have $Z^{*}=K^{*}$. Next, by the linearlization method about the equilibrium $\mathbf{E}^{*}$, let $y(t)=Y(t)-Y^{*}$ and $z(t)=Z(t)-Z^{*}$. Then, substitute $y$ and $z$ into (10). By using the Taylor series expansion, we have the linearlized system as

$$
\left[\begin{array}{c}
y^{\prime}(t) \\
z^{\prime}(t)
\end{array}\right]=\left[\begin{array}{cc}
\alpha\left(I_{Y}^{*}-S_{Y}^{*}\right) & \alpha I_{Z}^{*} \\
I_{Y}^{*} & -\beta
\end{array}\right]\left[\begin{array}{c}
y(t) \\
z(t)
\end{array}\right]+\left[\begin{array}{cc}
0 & 0 \\
0 & I_{Z}^{*}
\end{array}\right]\left[\begin{array}{c}
y(t-\tau) \\
z(t-\tau)
\end{array}\right]
$$

where $I_{Y}^{*}=\frac{\partial I}{\partial Y}\left(Y^{*}, Z^{*}\right), I_{Z}^{*}=\frac{\partial I}{\partial K}\left(Y^{*}, Z^{*}\right)$ and $S_{Y}^{*}=$ $\frac{d S}{d Y}\left(Y^{*}, Z^{*}\right)$

The Jacobian matrix at $\mathbf{E}^{*}$ is

$$
J\left(\mathbf{E}^{*}\right)=\left[\begin{array}{cc}
\alpha\left(I_{Y}^{*}-S_{Y}^{*}\right) & \alpha I_{Z}^{*} \\
I_{Y}^{*} & -\beta+e^{-\lambda \tau} I_{Z}^{*}
\end{array}\right] .
$$

Then, the characteristic equation is

$$
\begin{array}{r}
\lambda^{2}+\left(\beta-\alpha\left(I_{Y}^{*}-S_{Y}^{*}\right)-I_{Z}^{*} e^{-\lambda \tau}\right) \lambda+\alpha\left(\left(I_{Y}^{*}-S_{Y}^{*}\right)\right. \\
\left.\left(I_{Z}^{*} e^{-\lambda \tau}-\beta\right)-I_{Y}^{*} I_{Z}^{*}\right)=0 .
\end{array}
$$

Note that (12) is used to analyze stability of the equilibrium.

Theorem 2. For $\tau=0$, the equilibrium $\boldsymbol{E}^{*}=\left(Y^{*}, Z^{*}\right)$ of model (4) that satisfies conditions in (6)-(7) is locally asymptotically stable if and only if

$$
I_{Y}^{*}-S_{Y}^{*}<\min \left\{\frac{\beta-I_{Z}^{*}}{\alpha}, \frac{I_{Y}^{*} I_{Z}^{*}}{I_{Z}^{*}-\beta}\right\}
$$

Proof. From (12), it can be seen that

$$
I_{Z}^{*}=I_{K}^{*}
$$

Without time delay or $\tau=0$, the characteristic equation (12) is a quadratic equation of the form

$$
\begin{aligned}
& \lambda^{2}+\left(\beta-\alpha\left(I_{Y}^{*}-S_{Y}^{*}\right)-I_{Z}^{*}\right) \lambda \\
& \quad+\alpha\left(\left(I_{Y}^{*}-S_{Y}^{*}\right)\left(I_{Z}^{*}-\beta\right)-I_{Y}^{*} I_{Z}^{*}\right)=0 .
\end{aligned}
$$

Note that all roots $(\lambda)$ of the characteristic equation (14) have negative real part if and only if $B>0$ and $C>0$, where

$$
\begin{aligned}
& B=\beta-\alpha\left(I_{Y}^{*}-S_{Y}^{*}\right)-I_{Z}^{*}, \\
& C=\alpha\left(\left(I_{Y}^{*}-S_{Y}^{*}\right)\left(I_{Z}^{*}-\beta\right)-I_{Y}^{*} I_{Z}^{*}\right) .
\end{aligned}
$$

From $B>0$, we have that

$$
I_{Y}^{*}-S_{Y}^{*}<\frac{\beta-I_{Z}^{*}}{\alpha}
$$

From $C>0$, then we have

$$
I_{Y}^{*}-S_{Y}^{*}<\frac{I_{Y}^{*} I_{Z}^{*}}{I_{Z}^{*}-\beta}
$$

which is equivalent to

$$
I_{Y}^{*}-S_{Y}^{*}<\min \left\{\frac{\beta-I_{Z}^{*}}{\alpha}, \frac{I_{Y}^{*} I_{Z}^{*}}{I_{Z}^{*}-\beta}\right\} .
$$

This completes the proof of Theorem 2 .

In the case of positive delay $\tau>0$, we will firstly discuss the local stability of the positive equilibrium and then analyze the conditions for an occurrence of a Hopf bifurcation. Let the time delay $\tau$ be the bifurcation parameter. We will analyze the behaviors of model (4).

Because of the condition in (13), the characteristic equation (12) about the equilibrium $\mathbf{E}^{*}=\left(Y^{*}, K^{*}\right)$ is given by

$$
\begin{aligned}
& \lambda^{2}+\left(\beta-\alpha\left(I_{Y}^{*}-S_{Y}^{*}\right)-I_{K}^{*} e^{-\lambda \tau}\right) \lambda \\
& +\alpha\left(\left(I_{Y}^{*}-S_{Y}^{*}\right)\left(I_{K}^{*} e^{-\lambda \tau}-\beta\right)-I_{Y}^{*} I_{K}^{*}\right)=0 .
\end{aligned}
$$

We assume that $\lambda=i \omega$ (with $\omega>0$ ) is a purely imaginary root of (12) and separating the real part and the imaginary part. The real part of $\lambda$ satisfies

$$
\begin{array}{r}
-\omega^{2}+\left(-I_{Y}^{*} I_{K}^{*}-\beta\left(I_{Y}^{*}-S_{Y}^{*}\right)+\beta\right)=\omega I_{K}^{*} \cos (\omega \tau) \\
-\left(I_{Y}^{*}-S_{Y}^{*}\right) I_{K}^{*} \sin (\omega \tau),
\end{array}
$$

and the imaginary part is related to the condition

$$
\begin{array}{r}
\omega\left(-\alpha\left(I_{Y}^{*}-S_{Y}^{*}\right)+\beta\right)=\omega I_{K}^{*} \sin (\omega \tau) \\
+\left(I_{Y}^{*}-S_{Y}^{*}\right) I_{K}^{*} \cos (\omega \tau) .
\end{array}
$$

Solving (15) and (16) for $\cos (\omega \tau)$ and $\sin (\omega \tau)$, we have

$$
\begin{aligned}
& \cos (\omega \tau)=\frac{\left(\left(I_{K}^{*}+\beta\right) I_{Y}^{*}-S_{Y}^{*} \beta\right)\left(I_{Y}^{*}-S_{Y}^{*}\right) \alpha^{2}+\beta \omega^{2}}{\left(\left(I_{Y}^{*}-S_{Y}^{*}\right)^{2} \alpha^{2}+\omega^{2}\right) I_{K}^{*}}, \\
& \sin (\omega \tau)=-\frac{\omega\left(\left(I_{Y}^{*}-S_{Y}^{*}\right)^{2} \alpha^{2}+I_{Y}^{*} I_{K}^{*} \alpha+\omega^{2}\right)}{\left(\left(I_{Y}^{*}-S_{Y}^{*}\right)^{2} \alpha^{2}+\omega^{2}\right) I_{K}^{*}} .
\end{aligned}
$$

From (17), adding squares for both equations. We have the fourth-degree of polynomial equation as

$$
\omega^{4}+D \omega^{2}+E=0
$$

where

$$
D=\left(\left(I_{Y}^{*}-S_{Y}^{*}\right)^{2} \alpha^{2}+2 \alpha I_{Y}^{*} I_{K}^{*}-\left(I_{K}^{*}\right)^{2}+\beta^{2}\right),
$$

$E=\left(\left(I_{Y}^{*}-S_{Y}^{*}\right) \beta+I_{Y}^{*} I_{K}^{*}\right)^{2} \alpha^{2}-\alpha^{2}\left(I_{Y}^{*}-S_{Y}^{*}\right)^{2}\left(I_{K}^{*}\right)^{2}$ 
Lemma 1. Let $D$ and $E$ be defined in (19) and (20) respectively.

(i) If $D \geq 0$ and $E \geq 0$, then (18) has no positive roots. All roots of (18) have negative real parts for $\tau \geq 0$.

(ii) If $D<0$, then (18) has unique positive root $\omega_{0}$,

where

$$
\omega_{0}=\frac{\sqrt{2}}{2}\left(-D+\sqrt{D^{2}-4 E}\right)^{\frac{1}{2}},
$$

and there exists one sequence of critical values of $\tau$ given by

$$
\tau_{j}^{0}=\frac{1}{\omega_{0}} \arccos \left(\frac{\left(\left(I_{K}^{*}+\beta\right) I_{Y}^{*}-S_{Y}^{*} \beta\right)\left(I_{Y}^{*}-S_{Y}^{*}\right) \alpha^{2}+\beta \omega_{0}^{2}}{\left(\left(I_{Y}^{*}-S_{Y}^{*}\right)^{2}+\omega_{0}^{2}\right) I_{K}^{*}}\right)
$$$$
+\frac{2 \pi j}{\omega_{0}}, j \in \mathbb{N} . \text { The proof of Lemma } 2 \text { is completed. }
$$

In the same way, we have

$$
\left.\operatorname{Re}\left\{\left(\frac{d \lambda}{d \tau}\right)^{-1}\right\}\right|_{\tau=\tau_{j}^{0}}>0
$$

(iii) If $D<0, E>0$ and $D^{2}-4 E>0$ then (18) has

two positive roots, $\omega_{ \pm}$, where

$$
\omega_{ \pm}=\frac{\sqrt{2}}{2}\left(-D \pm \sqrt{D^{2}-4 E}\right)^{\frac{1}{2}}
$$

and there exist two sequences of critical values $\tau$, which are

$$
\begin{array}{r}
\tau_{j}^{ \pm}=\frac{1}{\omega_{ \pm}} \arccos \left(\frac{\left(\left(I_{K}^{*}+\beta\right) I_{Y}^{*}-S_{Y}^{*} \beta\right)\left(I_{Y}^{*}-S_{Y}^{*}\right) \alpha^{2}+\beta \omega_{ \pm}^{2}}{\left(\left(I_{Y}^{*}-S_{Y}^{*}\right)^{2}+\omega_{ \pm}^{2}\right) I_{K}^{*}}\right) \\
+\frac{2 \pi j}{\omega_{ \pm}}, j \in \mathbb{N} .
\end{array}
$$

Lemma 2. Let $\lambda(\tau)=\sigma(\tau)+i \omega(\tau)$ be the root of (12) and assume that $\sigma\left(\tau_{j}^{ \pm}\right)=0$ and $\omega\left(\tau_{j}^{ \pm}\right)=\omega^{ \pm}$. Thus

$$
\operatorname{Re}\left(\frac{d\left(\lambda\left(\tau_{j}^{+}\right)\right)}{d \tau}\right)>0, \text { and } \operatorname{Re}\left(\frac{d\left(\lambda\left(\tau_{j}^{-}\right)\right)}{d \tau}\right)<0 .
$$

Proof. Substitute $\lambda(\tau)$ into (12) and differentiate both sides of (12) with respect to $\tau$. The result is

$$
\begin{aligned}
\left(\frac{d \lambda}{d \tau}\right)^{-1}= & \frac{2 \lambda}{I_{K}^{*}\left(-\lambda+\alpha\left(I_{Y}^{*}-S_{Y}^{*}\right)\right) e^{-\lambda \tau} \lambda} \\
& +\frac{-I_{K}^{*}\left(-\tau \lambda+1+\tau\left(I_{Y}^{*}-S_{Y}^{*}\right) \alpha\right) e^{-\lambda \tau}}{I_{K}^{*}\left(-\lambda+\alpha\left(I_{Y}^{*}-S_{Y}^{*}\right)\right) e^{-\lambda \tau} \lambda} \\
& +\frac{-\lambda\left(I_{Y}^{*}-S_{Y}^{*}\right)+\beta}{I_{K}^{*}\left(-\lambda+\alpha\left(I_{Y}^{*}-S_{Y}^{*}\right)\right) e^{-\lambda \tau} \lambda} .
\end{aligned}
$$

Calculate for the real part, we have

$$
\begin{aligned}
\left.\operatorname{Re}\left\{\left(\frac{d \lambda}{d \tau}\right)^{-1}\right\}\right|_{\tau_{j}^{ \pm}} & =\frac{\left(I_{Y}^{*}-S_{Y}^{*}\right)^{2} \alpha^{2}+2 I_{Y}^{*} I_{K}^{*} \alpha+\beta^{2}}{\left(I_{K}^{*}\right)^{2}\left(\left(I_{Y}^{*}-S_{Y}^{*}\right)^{2} \alpha^{2}+\omega_{ \pm}^{2}\right)} \\
& =\frac{2 \omega_{ \pm}^{2}+D}{\left(I_{K}^{*}\right)^{2}\left(\left(I_{Y}^{*}-S_{Y}^{*}\right)^{2} \alpha^{2}+\omega_{ \pm}^{2}\right)} \\
& =\frac{ \pm \sqrt{D^{2}-4 E}}{\left(I_{K}^{*}\right)^{2}\left(\left(I_{Y}^{*}-S_{Y}^{*}\right)^{2} \alpha^{2}+\omega_{ \pm}^{2}\right)},
\end{aligned}
$$

where $D^{2}-4 E>0$ and $\left(I_{K}^{*}\right)^{2}\left(\left(I_{Y}^{*}-S_{Y}^{*}\right)^{2} \alpha^{2}+\omega_{ \pm}^{2}\right) \neq 0$. Then it can be seen that

$$
\left.\operatorname{Re}\left\{\left(\frac{d \lambda}{d \tau}\right)^{-1}\right\}\right|_{\tau=\tau_{j}^{+}}>0
$$

According to Lemma 1 and Lemma 2, we can conclude the main results for stability properties for the business equilibrium $\mathbf{E}^{*}$ of (4) as the following theorem.

Theorem 3. Let $\boldsymbol{E}^{*}$ be the equilibrium of (4), D and $E$ define in (19)-(20), respectively. If $\tau>0$, then the dynamics of (4) can be concluded as follows.

(i) If $D>0$ and $E>0$, then the equilibrium $\boldsymbol{E}^{*}$ is locally asymptotically stable when $\tau>0$.

(ii) If $D<0$, then the equilibrium $\boldsymbol{E}^{*}$ is locally asymptotically stable when $\tau \in\left(0, \tau_{0}^{+}\right)$, and it is unstable when $\tau>\tau_{0}^{+}$. Furthermore, the system (4) occurs a Hopf bifurcation when $\tau=\tau_{j}^{0}$, where $j \in \mathbb{N}$.

(iii) If $D<0, E>0$ and $D^{2}-4 E>0$, then the equilibrium $\boldsymbol{E}^{*}$ is locally asymptotically stable when $\tau \in\left[0, \tau_{0}^{+}\right) \cup\left(\tau_{0}^{-}, \tau_{1}^{+}\right) \cup\left(\tau_{1}^{-}, \tau_{2}^{+}\right) \cup \cdots \cup\left(\tau_{n-1}^{-}, \tau_{n}^{+}\right)$, where $n$ is a positive integer, and $\boldsymbol{E}^{*}$ is unstable, when $\tau=\left[\tau_{0}^{+}, \tau_{0}^{-}\right) \cup\left(\tau_{1}^{+}, \tau_{1}^{-}\right) \cup \cdots \cup\left(\tau_{n}^{+}, \tau_{n}^{-}\right) \cup$ $\left(\tau_{n}^{+},+\infty\right)$. Moreover, if $\tau=\tau_{j}^{ \pm}$, then (4) occurs a Hopf bifurcation at the equilibrium $\boldsymbol{E}^{*}$.

\section{Numerical Simulations}

In the numerical simulations, we apply the theoretical results in the previous section to verify the business cycle model with an expectation and a time delay. In case that $\tau=0$, let the investment function $I(Y, K)=\frac{e^{Y}}{1+e^{Y}}-q\left(\frac{e^{K}}{1+e^{K}}\right)$ and the saving function $S(Y)=\gamma Y$. We assume that the parameter values are $\alpha=2, q=0.3, \gamma=0.1$ and $\beta=0.1$. The equilibrium is $\mathbf{E}^{*}=(2.265,2.265)$, which is asymptotically stable by Theorem 2. The numerical results are shown in Figure 1. 


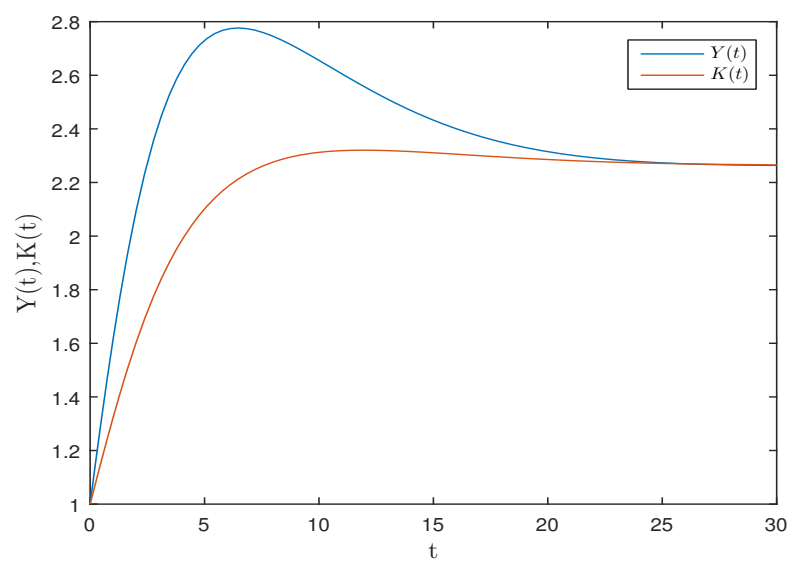

Figure 1. Solutions of (4) for $\mathbf{E}^{*}=(2.265,2.265)$, when $\tau=0$.

Next, change the parameter value $\beta=0.2$ and other parameter values are fixed to be the same. The equilibrium is changed to $\mathbf{E}^{*}=(3.922,1.961)$ and it is asymptotically stable. The results are shown in Figure 2.

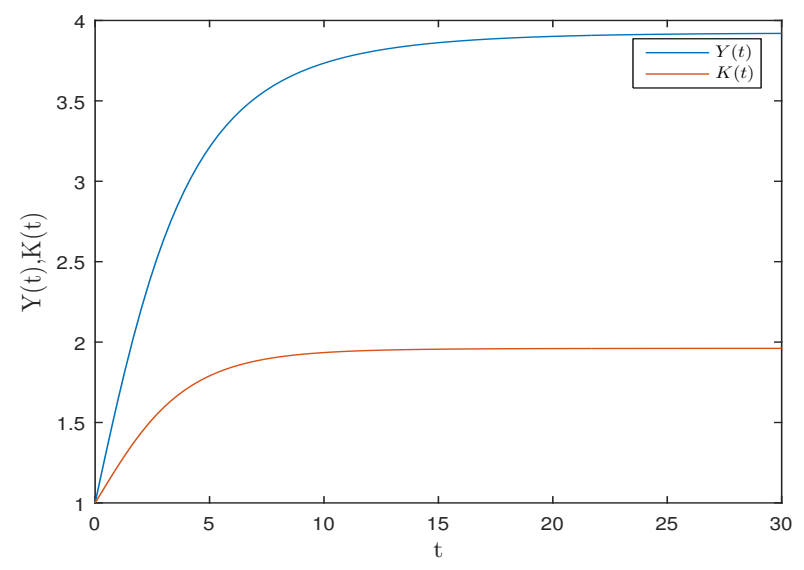

Figure 2. Solutions of (4) for $\mathbf{E}^{*}=(3.922,1.961)$, when $\tau=0$.

Furthermore, in case $\tau>0$, we show numerical simulations for two different investment functions:

$$
\begin{aligned}
& I_{1}(Y, K)=\frac{e^{Y}}{1+e^{Y}}-q\left(\frac{e^{K}}{1+e^{K}}\right) \\
& I_{2}(Y, K)=\frac{e^{Y}}{1+e^{Y}}-\frac{q K}{\sqrt{\varepsilon K^{2}+1}},
\end{aligned}
$$

where $q>0$ and $\varepsilon>0$.

For $I_{1}$, assume that $S(Y)=\gamma Y$, and parameter values are $\alpha=2, q=0.7, \gamma=0.2$, and $\beta=0.1$. Therefore, model (4) becomes

$$
\begin{aligned}
& \frac{d Y(t)}{d t}=2\left[\frac{e^{Y(t)}}{1+e^{Y(t)}}-0.7\left(\frac{e^{K(t+\tau)}}{1+e^{K(t+\tau)}}\right)-0.2 Y(t)\right], \\
& \frac{d K(t)}{d t}=\frac{e^{Y(t-\tau)}}{1+e^{Y(t-\tau)}}-0.7\left(\frac{e^{K(t-\tau)}}{1+e^{K(t-\tau)}}\right)-0.1 K(t) .
\end{aligned}
$$

The equilibrium of (23) is $\mathbf{E}^{*}=(1.090,0.545)$. By calculation, we have $\omega_{0}=0.346$ and $\tau_{0}=6.114$. From Theorem 3 , the equilibrium $\mathbf{E}^{*}$ is asymptotically stable when $0<\tau<\tau_{0}$ (see Figure 3). The Hopf bifurcation occurs when $\tau=\tau_{0}$. Therefore, if $\tau>\tau_{0}$, then the solutions of (23) are periodic. The results in this case are shown in Figure 4.
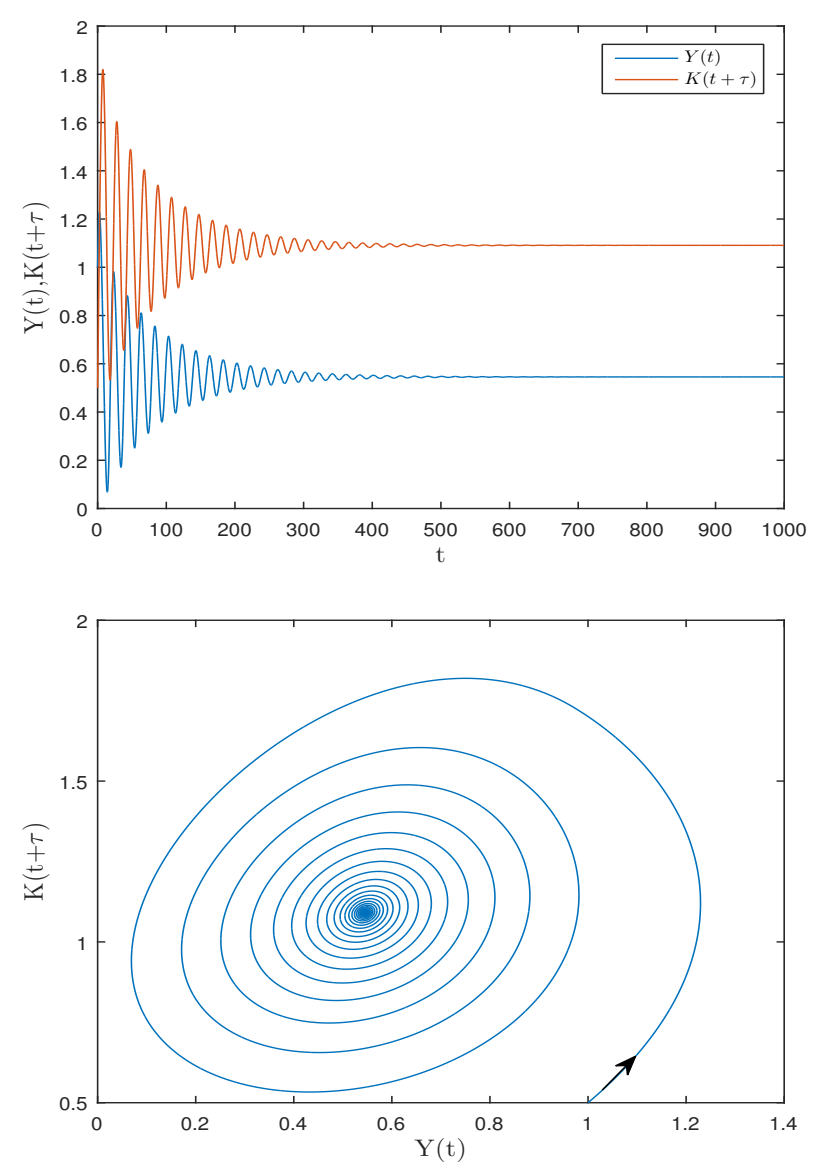

Figure 3. The asymptotic stability of $\mathbf{E}^{*}=(1.090,0.545)$ for $\tau=6.014<\tau_{0}$.

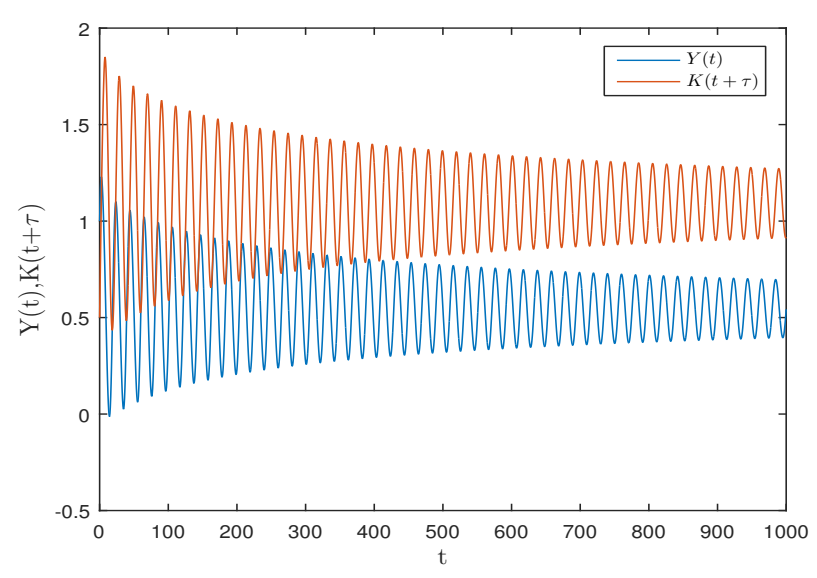




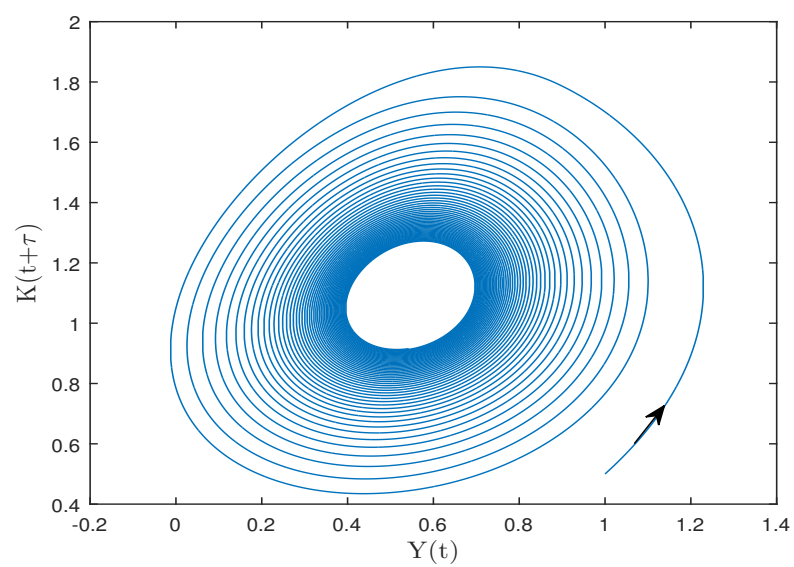

Figure 4. The periodic solutions of (23) for $\tau=6.614>\tau_{0}$.

The second investment function $I_{2}$ is used in [12]. Let the parameter values be $\alpha=1, q=0.7, \varepsilon=0.1, \gamma=0.6$ and $\beta=0.1$, then model (4) becomes

$\frac{d Y(t)}{d t}=1\left[\frac{e^{Y(t)}}{1+e^{Y}(t)}-\frac{0.7 K(t+\tau)}{\sqrt{0.1 K(t+\tau)^{2}+1}}-0.6 K(t)\right]$, $\frac{d K(t)}{d t}=\frac{e^{Y(t-\tau)}}{1+e^{Y(t-\tau)}}-\frac{0.7 K(t-\tau)}{\sqrt{0.1 K(t-\tau)^{2}+1}}-0.1 K(t)$.

The equilibrium of (24) is $\mathbf{E}^{*}=(0.112,0.673)$. After the calculation, we have $\omega_{0}=0.802$ and $\tau_{0}=2.274$. The equilibrium $\mathbf{E}^{*}$ is asymptotically stable when $\tau \in\left[\tau, \tau_{0}\right)$ according to Theorem 3. The results in this case are shown in Figure 5.

Next, model (4) occurs a Hopf bifurcation if $\tau=\tau_{0}$ and the solution of (4) is a family of periodic solutions around the equilibrium $\mathbf{E}^{*}$ when $\tau>\tau_{0}$ as shown in Figure 6.

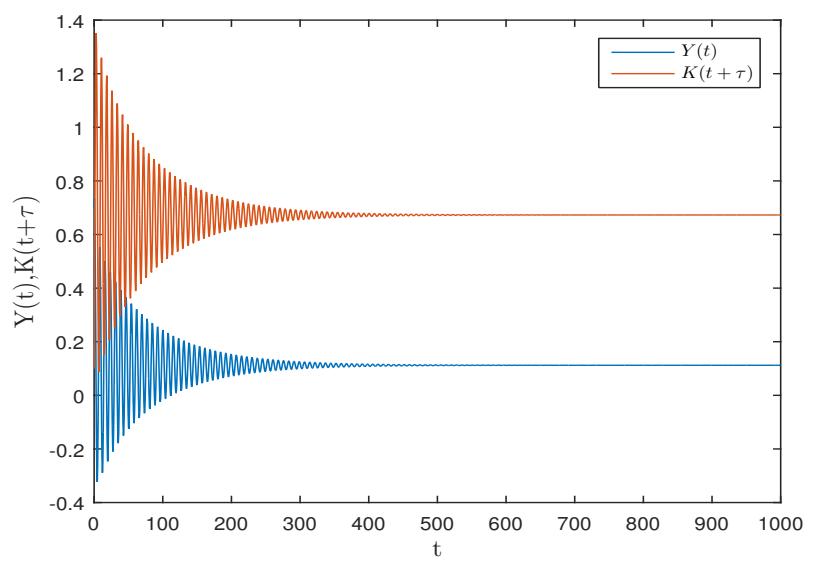

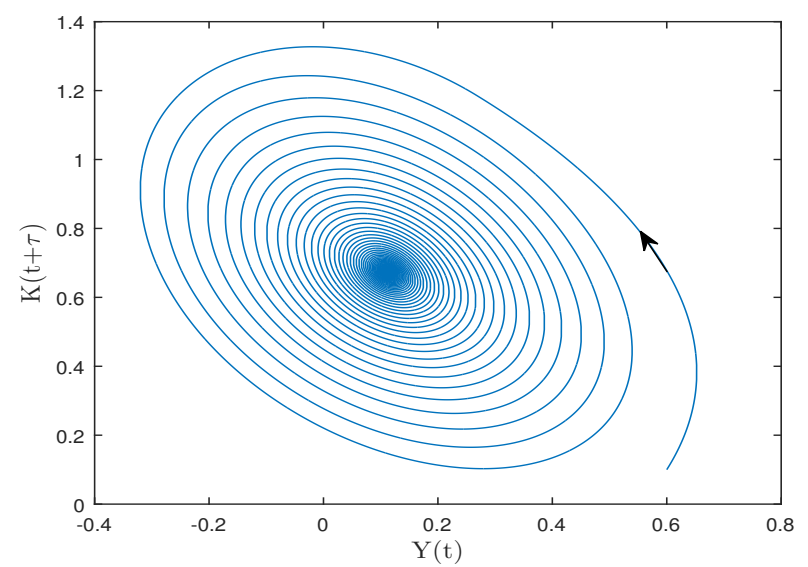

Figure 5. The asymptotic stability of $\mathbf{E}^{*}=(0.112,0.673)$ for $\tau=2.200<\tau_{0}$.
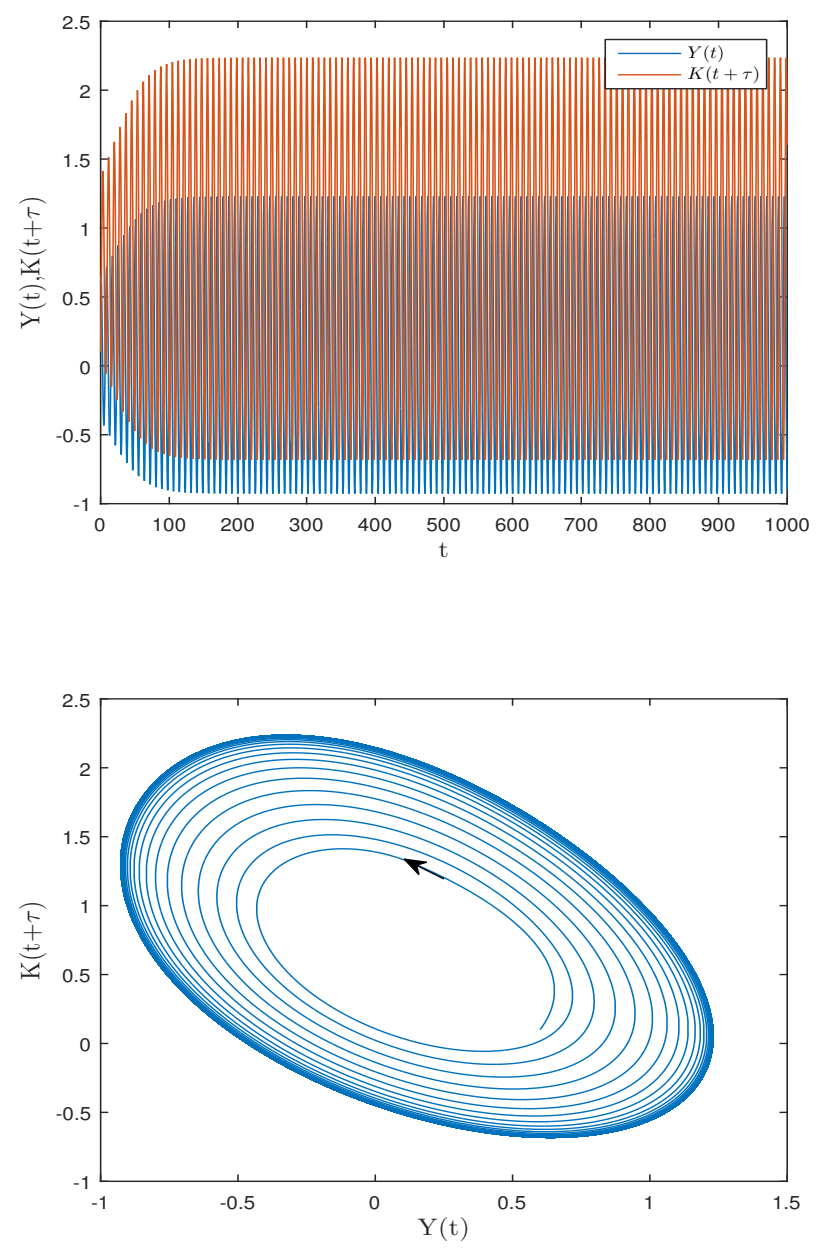

Figure 6. The periodic solutions of (24) for $\tau=2.524>\tau_{0}$.

\section{Conclusions}

In this work, the business cycle model with an expectation and lag time to investment has been studied. The model is analyzed by using advantages of mathematical theorems, which 
are the local stability theorem and the Hopf bifurcation theorem. The studied model represents the relation between the rates of changes for the gross product and the capital stock, with general investment and saving functions.

The main aim for this work is to study effects of the time delay or the lag time for investment to the occurrence of a business cycle in the model. The results show that if there is no waiting time for investment, then there is no cycle between the gross product and the capital stock. The solutions in this case are asymptotically stable, which means that all solutions converge to the business equilibrium when time increases. On the contrary, for a sufficient large of time delay, or decision time to investment, there exists a business cycle which shows periodicity of solutions between the gross product and the capital stock. The results from mathematical analysis are also illustrated by the numerical simulations. We have already showed that the analytical results are compatible with the numerical simulations.

\section{Acknowledgements}

We would like to thank the referees for valuable comments and suggestions on the manuscript, which help us to improve the paper. Moreover, we sincerely thank to Department of Mathematics and Graduate College, King Mongkut's University North Bangkok (Thailand) for the financial support.

\section{REFERENCES}

[1] M. Kalecki, A macrodynamic theory of business cycles, JSTOR, Econometrica, Journal of the Econometric Society, 327-344, 1935.

[2] N. Kaldor, A model of the trade cycle, JSTOR, The Economic Journal, 78-92, 1940.

[3] W. W. Chang, D. J. Smyth, The existence and persistence of cycles in a non-linear model: Kaldor's 1940 model re-examined, JSTOR, The Review of Economic Studies, Vol. 38, No. 1, 37-44, 1971 .

[4] J. Grasman, J. J. Wentzel, Co-existence of a limit cycle and an equilibrium in Kaldor's business cycle model and its consequences, Elsevier, Journal of Economic Behavior and Organization, Vol. 24, No. 3, 369-377, 1994.

[5] E. Kunnawuttipreechachan, Stability of a numerical discretization scheme for the SIS epidemic model with a delay, IAENG, Proceedings of the World Congress on Engineering, London, Vol. 3, 1923-1930, 2010
[6] S. Udomchalermpat, S. Koonpraseart, E. Kunnawuttipreechachan, Dynamics of the generalized tumor-virotherapy model with time delay effect, IAENG, Engineering Letters,

Vol. 28, No. 3, 2020.

[7] S. Udomchalermpat, S. Koonpraseart, E. Kunnawuttipreechachan, The effect of obesity and cancer stem cells in tumor model with time delay, COMPUSOFT, An International Journal of Advanced Computer Technology, Vol. 9, No. 4, 3633-3641, 2020.

[8] L. Lleshaj, A. Korbi, Portfolio selection and VaR estimation: evidence from western Balkan countries, Universal Journal of Accounting and Finance, Vol. 8, No. 4, 92-102, 2020.

[9] H. Yang, M. Meysami, A. Meisami, A rising market and capital structure decisions, Universal Journal of Accounting and Finance, Vol. 5, No. 4, 2017.

[10] A. Krawiec, M. Szydlowski, The Kaldor-Kalecki business cycle model, Springer, Annals of Operations Research, Vol. 89 , 89-100, 1999.

[11] X. P. Wu, L. Wang, A Krawiec-Szydlowski model of business cycles with a time delay in capital stock, Oxford University Press, The IMA Journal of Applied Mathematics, Vol. 79, No. 3, 571-599, 2014.

[12] D. Riad, K. Hattaf, N. Yousfi, Dynamics of a delayed business cycle model with general investment function, Elsevier, Chaos, Solitons \& Fractals, Vol. 85, 110-119, 2016.

[13] K. Hattaf, D. Riad, N. Yousfi, A generalized business cycle model with delays in gross product and capital stock, Elsevier, Chaos, Solitons \& Fractals, Vol. 98, 31-37, 2017.

[14] C. Jianzhi, S. Hongyan. Bifurcation analysis for the Kaldor-Kalecki model with two delays, SpringerOpen, Advances in Difference Equations, Vol. 2019, No. 1, 1-27, 2019.

[15] A. Kaddar, H. T. Alaoui, Hopf bifurcation analysis in a delayed Kaldor-Kalecki model of business cycle, Nonlinear Analysis: Modelling and Control, Vol. 13, No. 4, 439-449, 2008.

[16] Y. Zheng, S. Liu, A dynamic analysis of the business cycle model with a fixed-time delay, Quantitative Finance and Economics, Vol. 1, No. 2, 174-185, 2017. 